\title{
Exact Axisymmetric Thermal Analysis of Functionally Graded Disks with Continuously Hyperbolically Varying Thickness
}

\author{
Vebil Y1ldırım* \\ *Department of Mechanical Engineering, Faculty of Engineering and Architecture, University of Çukurova, Adana \\ (vebil@cu.edu.tr, ORCID: 0000-0001-9955-8423)
}

Received: 15.02.2020 Accepted: 18.09.2020

\begin{abstract}
An exact thermal analysis of radially functionally graded (FG) disks with continuously varying thickness is performed by steady-state 1-D Fourier heat conduction equation at specific surface temperatures. By employing a simple-power material grading pattern together with the convergent/divergent hyperbolic disk profiles, the differential equation is obtained in the form of Euler-Cauchy type. Analytical solution of the differential equation gives the temperature field and heat flux distributions in the radial direction in a closed form. A numerical study is conducted to visualize both the temperature and heat flux variations with respect to the disk profile parameter for hyperbolic disks made of SUS-304 $/ \mathrm{ZrO}_{2}$ (Stainless steel/Zirconium oxide) metalceramic pairs. Those exact expressions are also used to study parametrically the effects of both the inhomogeneity and profile parameters on the temperature field of the disks made of hypothetic FG metal-ceramic pairs. It is revealed that heat conduction behavior of such disks is strictly affected from the variation of both inhomogeneity and disk profile parameters.
\end{abstract}

Keywords. Thermal analysis, functionally graded, exact solution, variable thickness, hyperbolic disk.

\section{Introduction}

Disks are fundamental structural components of machines exploited in many industrial sectors such as aviation, automobile, aerospace, agribusiness, power, computer, and petroleum to be used for many purposes. They may be functioned as a stationary/rotating circular annulus or as a rotating disk attaching a shaft at its center. Their thickness may vary along the radial direction linearly, hyperbolically, parabolically, or so on and so forth. It may be made of any kind of conventional or advanced materials.

Disks may be mainly constructed to operate in high temperature environments like turbine rotors, flywheels and gears. They may also be subjected to friction-induced heat in the operating environment. In these cases the thermo-elastic analyses gain a great importance in the design of such structures. In the thermal-related analyses, the first step is to determine truly the thermal analysis that is the temperature distribution along the radial coordinate of the disks.
It may be noted that the temperature field and heat flux distribution are the same for both cylinders and disks with uniform thickness. However, a disk with variable thickness offers different temperature and heat flux profiles than hollow cylinders. Some works just on the thermal-related analyses of either uniform or varying-thickness disks will be mentioned below.

Thermal-related analyses of uniform disks made of an isotropic and homogeneous materials have paid much attention than disks having varying section properties and made of advanced composite materials [1-10]. From those, Güven and Altay [1] investigated the elastic-plastic stress distribution of a solid disk due to nonuniform heat source under external pressure. They presented an exact solution based on the usual assumptions of plane stress, Tresca's yield condition, its associated flow rule and linear strain hardening material behaviour. Jahed and Shirazi [2] researched loading and residual stresses, and associated strains and displacements in thermoplastic uniform rotating disks at elevated 
temperatures. Kulkarni and Deshmukh [4] studied the thermal stresses in a thick annular disk under steady temperature field. Based on a 3D numerical calculations, Genç et al. [5] worked on the effects of a moving heat source in the radial direction on a rotating hollow steel rotating disk heated from its one side surface under stagnant ambient conditions. Nejad and Afshin [6] offered an analytical solution of transient thermoelastic behaviors of rotating pressurized disks subjected to arbitrary boundary and initial conditions. Rattan et al. [7] investigated the steady-state creep behavior of thermally graded isotropic uniform disk, made of aluminum matrix reinforced with silicon carbide particulate, rotating at elevated temperature. Kaur et al. [8] applied Seth's transition theory to the problems of thickness variation parameter in a thin rotating disk by finite deformation. Neither the yield criterion nor the associated flow rule was assumed in their study. Kaur et al. [8] observed that thermal effect in the disk increase the value of circumferential stress at the internal surface and radial stresses at the external surface for compressible as compare to incompressible material. Based on a variational principle considering the radial displacement field as unknown, Nayak and Saha [9] evaluated the influence of thermo-mechanical loading on stresses and deformation states in a rotating disk with varying thicknesses. They considered different disk geometries as well as temperature distribution profiles to calculate the limit angular speed of the disks under thermomechanical loading. Y1ldırım [10] offered a consistent and an all-in-one study for the analytically determination of heatinduced, pressure-induced, and centrifugal force-induced axisymmetric exact deformation and stresses in a thick-walled spherical vessel, a cylindrical vessel, and a uniform disk at specified constant surface temperatures and at a constant angular velocity. Yildırım [10] included both the inner and outer pressures in the formulation of annular structures made of an isotropic and homogeneous linear elastic material. For disks, three different boundary conditions were taken into account to consider mechanical engineering applications in the study. Yildırım [10] also presented closed-form compact formulas under the same notations for all types of annular structures to the readers.

There are relatively less study on the thermal-related analyses of isotropic and homogeneous disks with varying thickness. By using hyper-geometric differential equation in terms of radial displacement, Vivio and Vullo [11] and Vullo and Vivio [12] introduced an analytical procedure for evaluation of elastic stresses and strains in rotating conical disks and in non-linear variable thickness rotating disks made of an isotropic and homogeneous material, either solid or annular, subjected to thermal loads. Nie and Batra [13] studied axisymmetric stresses in a rotating disk made of a rubber-like material that was modeled as isotropic, linear thermoelastic, and incompressible. In the presence of linear thermal gradient, Garg et al. [14] analyzed the steady state creep in a rotating disk having linearly varying thickness and made of composite containing silicon carbide particles $(\mathrm{SiC})$ in a matrix of pure aluminium. Çetin et. Al. [15] studied analytically an elastic stress analysis of annular isotropic and homogeneous bimaterial hyperbolic disks subjected to the mechanical and thermo-mechanical loads.
Thermal-related analyses of especially uniform disks made of anisotropic materials are other investigation themes. From those, Sayman [16] investigated a thermal stress analysis on an aluminum metal-matrix orthotropic composite uniform disk. Çallıoğlu et al. [17] dealt with the thermal stress analysis of a curvilinearly orthotropic rotating annular uniform disk under internal and external pressures. They used parobolically varying temperature distribution from the inner surface to the outer surface. As a continuation of this study, Çallıoğlu [18] considered uniform and linearly increasing and decreasing temperature profiles in his another study. Based on the finite element method, Şen and Sayer [19] investigated numerically elasto-plastic thermal stresses in a thermoplastic composite uniform disk that is reinforced by steel fibers, curvilinearly. Altan et al. [20] examined the thermo-elastic stress of a metal-matrix composite uniform disk under parobolically varying thermal loads. Mohammadi et al. [21] analyzed a composite rotating uniform disk under internal and external pressure subjected to a polynomial temperature distribution. Mustafa et al. [22] worked on thermal analysis of orthotropic fins. Kansal and Parvez [23] dealt with the stress analysis on orthotropic graded rotating annular uniform disks subjected to temperature distributions parabolically decreasing with radius. Lately, Stampouloglou and Theotokoglou [24] analyzed the radially nonhomogeneous thermoelastic axisymmetric problem in a hollow isotropic circular cylinder with a radially varying shear modulus and a constant Poisson's ratio, without internal and external loads, under on a radius depending temperature field.

Functionally graded materials are one of the types of advanced composite materials. In recent years functionally graded metal-ceramic composites gain considerable attention due to their attractive properties such as heat resisting, erosion and corrosion resistant, and have high fracture toughness. In the disk design, functionally graded materials are especially used for both increase of the critical speeds of the disk and also dissipation of the heat due to friction. It is also used to improve the heat transfer for fins [25-26].

As may be guessed effortlessly, most of the thermal-related studies on the disks made of functionally graded materials were conducted with disks having uniform thicknesses. Bayat et. al. [27] studied the thermoelastic analysis of a rotating disk made of functionally graded materials for small and large deformations. Kordkheili and Naghdabadi [28] presented semi-analytical thermo elastic solutions for hollow and solid rotating axisymmetric uniform disks made of functionally graded materials under plane stress condition. Arani et. Al. [29] investigated thermo-piezo-magnetic behavior of a functionally graded piezo-magnetic rotating uniform disk, under mechanical and thermal loads. By employing the finite element analysis, Afsar and Go [30] studied the thermoelastic analysis of a thin circular rotating uniform FGM disk. Peng and $\mathrm{Li}$ [31] examined analytically the analysis of thermoelastic problem of a rotating functionally graded hollow circular disk having varying material properties along the radial direction. They considered a boundary value problem having reduced to a Fredholm integral equation. Kurşun et al. [32] studied analytically the elastic stress analysis of annular disks made of functionally graded materials (FGMs) subjected to both uniform pressures on the 
inner surface and a linearly decreasing temperature distribution. The elasticity modulus and thermal expansion coefficient of the disks are assumed to vary radially according to power law functions and Poisson's ratio was kept constant [32]. By employing a finite volume method, Gong et al. [33] examined the steady thermoelastic analysis of the functionally graded circular rotating disk under different conditions. They showed that the least square method achieves better performances than the Gaussian method but least square method costs slightly more iteration and computer memory under different mesh types. The effects of thickness, material properties, reference temperature and temperature difference between the inner and outer surfaces on the thermoelastic performance of the disk have been studied in Gong et al.'s [33] study. Gonczi and Ecsedi [34] analyzed a thermoelastic boundary value problem of a hollow circular disk, made of functionally graded materials with arbitrary gradient, under a steady-state temperature distribution. Recently, Yıldırım [35] analytically investigated thermomechanical attributes of a power-law graded uniform mounted disk with or without rigid casing under individual and combined centrifugal and steadystate thermal loads within the framework of axisymmetric infinitesimal plane-stress elasticity theory. Two parametric studies in [35] were conducted by both hypothetically and physically chosen metal-ceramic pairs namely nickel-silicon nitride $\left(\mathrm{Ni}-\mathrm{Si}_{3} \mathrm{~N}_{4}\right)$, aluminum-aluminum oxide $\left(\mathrm{Al}-\mathrm{Al}_{2} \mathrm{O}_{3}\right)$, and stainless steel-zirconium oxide (SUS304-Z $\mathrm{rO}_{2}$ ).

Unfortunately, the number of studies on the thermal/thermal related analysis of variable disks are not enough. Chiba [36] evaluated the second-order statistics (i.e. mean and standard deviation) of the temperature and thermal stresses in an axisymmetrically heated functionally graded annular disk of variable thickness with spatially random heat transfer coefficients on the major surfaces of the disk. Chiba [36] assumed the annular disk is to be a multilayered one with stepwise thickness variation, where each layer is assumed to have constant deterministic material properties. Bayat et al. [37] considered a rotating functionally graded disk with either parabolic or hyperbolic thickness under a steady temperature field. The disk is assumed to be composed of sub-disks of uniform thicknesses. In another study, Bayat et. al [38] analyzed the thermoelastic bending of FGM rotating disks based on the first order shear deformation theory. Bayat et al. [39] studied on a thermo elastic analysis for axisymmetric rotating disks made of temperature-dependent power-law functionally graded material (FGM) with variable thickness. They assumed that the temperature field is to be uniformly distributed over the disk surface and varied in the radial direction. Bayat et al. [39] presented semi-analytical solutions for the displacement field for solid/annular disks under freefree and fixed-free boundary conditions. They showed the crucial role of the temperature-dependent properties in a high temperature environment. Bayat et al. [39] also showed that a functionally graded rotating disk with concave thickness profile can work more efficiently than the one with uniform thickness irrespective of whether the material properties are assumed to be temperature-dependent or temperatureindependent. Bayat et al. [40] also offered exact elastic solutions for axisymmetric variable-thickness hollow rotating disks with heat source made of functionally power-law-graded (FG) materials under free-free and fixed-free boundary conditions. They showed that the temperature distribution in a hyperbolic disk is the smallest compared with other thickness profiles. A hyperbolic-convergent FG disk has smaller stresses because of thermal load compared with the uniform disk. Arani et al. [41] suggested a semi-analytical solution for magneto-thermo-elastic problem in FGM hollow rotating disks with variable thickness placed in uniform magnetic and thermal fields. Damircheli and Azadi [42] carried out thermal and mechanical stress analyses of a rotating disk having either parabolically or hyperbolically varying thicknesses made of functionally graded material with variable thickness by using finite element method (FEM). By using a 2D plane stress analysis and assuming a power form temperature distribution of over the disk with the higher temperature at the outer surface, Hassani et al. [43] obtained stress and strains of rotating disks with non-uniform thickness and material properties subjected to thermo-elastic loading under different boundary conditions. They used semi-exact methods of Liao's homotopy analysis method (HAM), Adomian's decomposition method and He's variational iteration method (VIM). Hassani et al. [43] compared the results of those three methods with Runge-Kutta's results. They suggested the implementation of the proposed methods to provide accurate solution for a complicated case with no exact solution. Tütüncü and Temel [44] studied numerically the thermoelastic analysis of functionally graded rotating disks of variable thickness. Golmakani [45] scrutinized large deflection thermoelastic analysis of functionally graded solid and hollow rotating axisymmetric disk with uniform and variable thickness subjected to thermo-mechanical loading. Kurşun and Topçu [46] handled the elastic stress analysis of a hollow uniform disk with variable thickness made of functionally graded materials under linearly increasing temperature distribution. Mahdavi et al. [47] worked on the thermoplastic analysis of FG rotating hyperbolic disks by dividing the domain into some finite sub-domains in the radial direction, in which the properties are assumed to be constant. Recently, Jabbari et. al. [48] presented a thermoelastic analysis of rotating disks with different thickness profiles made of powergraded and exponential-graded materials subjected to internal pressure. They verified the results with finite element method.

The broad literature survey mentioned above showed that the realm of the thermal-related analyses of such disks having varying thicknesses need further works. As stated above, an accurate solution to the temperature field in the structure is a crucial first step to study in an appropriate manner the thermalrelated analyses. As the bare bones of the advanced thermalrelated analyses, in the present study just the thermal analysis of disks with hyperbolic profile is handled to observe both temperature field and heat flux variations through the radial direction. The heat conduction equation is first derived for disks with varying sections, and then solved analytically for simple-power graded disks having convergent/divergent hyperbolic thickness profiles (Fig 1).

Formulas are tested for a simple power graded physical metal-ceramic pair, namely SUS-304/ZrO2, at first place. Then, a parametric study is conducted for a hypothetically functionally graded material by considering the variations of both inhomogeneity and thickness profile parameters along 
the radial coordinate. Results are illustrated in both graphical and tabular forms.

\section{DIVERGENT HYPERBOLIC $/ \mathrm{m}=0.5$}

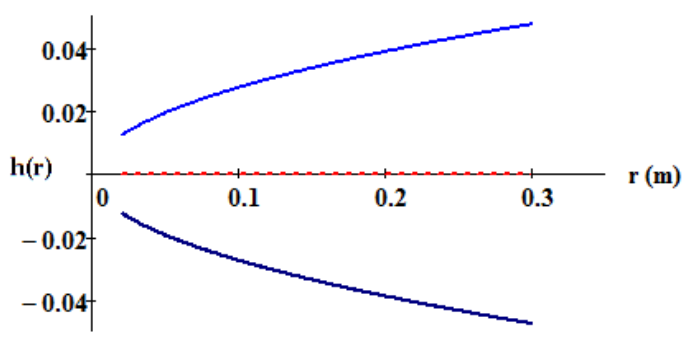

CONVERGENT HYPERBOLIC $/ \mathrm{m}=-0.5$

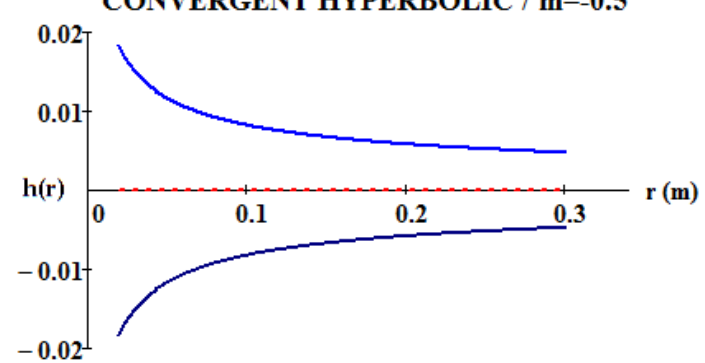

Fig. 1. Some convergent and divergent disk profiles, $h(r)=h_{a}(r / a)^{m}, \mathrm{a}=0.03 \mathrm{~m}, \mathrm{~b}=0.3 \mathrm{~m}$.

\section{Formulation and Solution}

The rate of the heat flux, $\boldsymbol{q}$, in a solid object is proportional to the temperature gradient, $\nabla T$. The Fourier law governing the heat transfer by conduction is

$$
\boldsymbol{q}=-k \nabla T=-k \operatorname{grad}(T)
$$

where $k$ is the thermal conductivity. Temperature gradient is given in cylindrical coordinates, $T(r, \theta, z)$, by

$$
\nabla T=\frac{\partial T}{\partial r} \boldsymbol{e}_{r}+\frac{1}{r} \frac{\partial T}{\partial \theta} \boldsymbol{e}_{\theta}+\frac{\partial T}{\partial z} \boldsymbol{e}_{z}
$$

where $\left(\boldsymbol{e}_{\boldsymbol{r}}, \boldsymbol{e}_{\theta}, \boldsymbol{e}_{z}\right)$ are unit vectors in cylindrical coordinates, $r$ is the radial coordinate, $\theta$ is the circumferential direction. The heat conduction equation may be written as follows by using the first law of thermodynamics.

$$
\rho c_{p} \frac{\partial T}{\partial t}+\operatorname{div}(\boldsymbol{q})=\dot{q}_{g e n}
$$

where $\rho$ is the density, and $c_{p}$ is the specific heat capacity,

$\dot{q}_{\text {gen }}$ is the heat generation per unit volume. Using Eq. (1) the following may be written for the divergence of the heat flux as

$$
\operatorname{div}(\boldsymbol{q})=\nabla \bullet \boldsymbol{q}=-k(\nabla \bullet \nabla T)=-k \Delta T=-k \nabla^{2} T
$$

By assuming that there is no heat generation in the structure, $\dot{q}_{g e n}=0$, and the steady state case $\left(\frac{\partial T}{\partial t}=0\right)$ exists, substitution of Eq. (4) into the heat conduction equation (3) gives the Laplacian of the temperature as follows.

$$
k \nabla^{2} T=0
$$

where Laplacian of the temperature, that is the divergence of the gradient of the temperature is derived in cylindrical coordinates as

$$
\nabla^{2} T=\frac{1}{r} \frac{\partial}{\partial r}\left(r \frac{\partial T}{\partial r}\right)+\frac{1}{r^{2}} \frac{\partial^{2} T}{\partial \theta^{2}}+\frac{\partial^{2} T}{\partial z^{2}}
$$

Combination of Eqs. (5) and (6) results a differential equation governing steady state $3-\mathrm{D}$ temperature distribution for solids having uniform thickness and constant thermal conductivity without heat generation.

For axisymmetric problems (derivatives with respect to the circumferential and z-directions are all zero) in polar coordinates, by taking into consideration of non-uniformity trait of both the thermal conductivity and the thickness profile in the radial direction, Eq. (5) takes the form of

$$
\frac{1}{r h(r)} \frac{d}{d r}\left(r k(r) h(r) \frac{d T(r)}{d r}\right)=0
$$

By using prime symbol for derivatives with respect to the radial coordinate, the above may also be written in the following form

$$
T^{\prime \prime}(r)+T^{\prime}(r)\left(\frac{1}{r}+\frac{k^{\prime}(r)}{k(r)}+\frac{h^{\prime}(r)}{h(r)}\right)=0
$$

In the above equation, the material grading pattern and the disk thickness profile may be chosen arbitrarily. Solution technique to be used strictly depends on the material grading pattern considered and disk profile type. Some very limited grading rules such as a simple power together with a hyperbolic disk profile permit to get differential equations with constant coefficients and offer analytical solutions. For general grading patterns and different disk profiles such as parabolic disk profile, Eq. (8) turns into a differential equation with variable coefficients. In such a case, in the analysis with general material grading patterns and disk profiles, it is necessary to use numerical techniques in the solution process. Finally all types of boundary conditions such as Dirichlet's, Neumann's, Robin's and mixed boundary conditions may be applied to the solution of those equations.

Bayat et al. [38], Golmakani [45], and Jabbari et al. [48] also offered the following solution to Eq. (8), $r k(r) h(r) \frac{d T(r)}{d r}=c_{1}$, for disks having arbitrary thickness and inhomogeneity profiles. 


$$
T(r)-T_{i}=c_{1} \int_{r_{i}}^{r} \frac{d r}{r h(r) k(r)}
$$

where

$$
c_{1}=\frac{T_{o}-T_{i}}{\int_{r_{i}}^{r_{o}} \frac{d r}{r h(r) k(r)}}
$$

As a consequence, the distribution of the temperature along the radial coordinate becomes $[38,45,48]$

$$
T(r)=T_{i}\left(1-\frac{\int_{r_{i}}^{r} \frac{d r}{r h(r) k(r)}}{r_{r_{i}} \frac{d r}{r h(r) k(r)}}\right)+T_{o}\left(\frac{\int_{r_{i}}^{r} \frac{d r}{r h(r) k(r)}}{\int_{r_{i}}^{r} \frac{d r}{r h(r) k(r)}}\right)
$$

where $r_{i}=a, r_{o}=b, T_{i}=T_{a}$, and $T_{o}=T_{b}$.

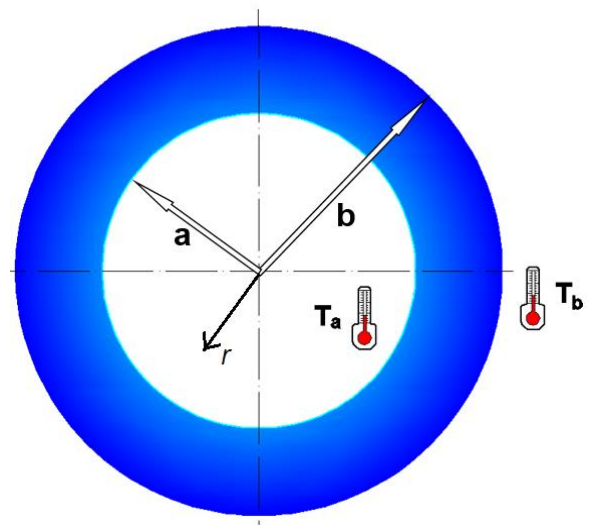

Fig. 2. The top-view of the disk

To achieve an exact solution to Eq. (8), in the present study, it is assumed that the thickness of the disk is changed according to the following hyperbolic disk profile

$$
h(r)=h_{a}\left(\frac{r}{a}\right)^{m}
$$

and the thermal conductivity is to be changed in radial direction, between the inner and outer surfaces, with the following function.

$$
k(r)=k_{a}\left(\frac{r}{a}\right)^{\gamma}
$$

In Eqs. (12) and (13), $a$ denotes the inner radius of the disk, $m$ is the profile parameter, and $\gamma$ is the inhomogeneity index (Fig. 2). The subscript $a$ illustrates the value of the related quantity at the inner surface. Denoting the outer radius of the disk by $b$, inhomogeneity index is determined by

$$
\gamma=\frac{\ln \left(\frac{k_{a}}{k_{b}}\right)}{\ln \left(\frac{a}{b}\right)}
$$

Here, it is assumed that at the inner surface is to be full with material-a, and the outer surface is to be full with material-b. Between the inner and outer surface, the material property of the functional mixture of two materials will obey Eq. (13).

Determination of the numerical value of the disk profile parameter is left to the user's choice. Its positive values offer a divergent disk profile (Fig. 1). The convergent hyperbolic disk profile is obtained by using negative profile parameters. Theoretically, the profile parameter may be greater than unit. However, it is also possible to obtain some unfeasible disk profiles for such higher values of the profile parameter. Before an analysis, the author recommends the visualization of the disk profile to avoid from handling an unreasonable disk profile.

Under the assumptions defined in Eqs. (12) and (13), Eq. (8) to be considered in the present study turns into the following

$$
T^{\prime \prime}(r)+\left(\frac{1}{r}+\frac{m}{r}+\frac{\gamma}{r}\right) T^{\prime}(r)=0
$$

Equation (15), incorporates both the variation of thermal conductivity and disk profile along the radial direction. Solution of Eq. (15) which is an Euler-Cauchy type differential equation with constant coefficients is given by

$$
T(r)=C_{1} r^{\mu_{1}}+C_{2} r^{\mu_{2}}
$$

where $\mu_{1}$ and $\mu_{2}$ are the characteristic roots of the differential equation, $C_{1}$ and $C_{2}$ are integration constants. In the present study the first kind boundary conditions (Dirichlet) are considered.

$$
T(a)=T_{a} ; T(b)=T_{b}
$$

In the above, $T_{a}$ and $T_{b}$ are the temperatures at the inner and outer surfaces, respectively. Considering Eq. (16) and boundary conditions in Eq. (17), the closed form solutions for each homogeneous/inhomogeneous material types and uniform/hyperbolic disks are presented in Tables 1 and 2 in compact forms.

\section{Examples}

As a first example a metal-ceramic pair is chosen as SUS$304 / \mathrm{ZrO}_{2}$ (Stainless steel/Zirconium oxide). Geometrical and material properties together with boundary conditions of the disk are assumed to be

$$
\begin{gathered}
a=0.03 m ; b=0.3 m ; h_{a}=a \\
T_{a}=100^{\circ} C ; T_{b}=20^{\circ} C
\end{gathered}
$$




$$
\begin{gathered}
k_{S U S-304}=15.379(\mathrm{~W} / \mathrm{mK}) ; k_{\mathrm{ZrO}_{2}}=1.78(\mathrm{~W} / \mathrm{mK}) ; \\
\gamma=-3.11101(\text { Eq. } 14)
\end{gathered}
$$

To handle a parametric study disk profile parameters are determined in the intervals: $m= \pm 0.25, \pm 0.50, \pm 0.75, \pm 1.00$.

Table 1. Differential equations and their solutions for uniform $\operatorname{disks}\left\{k(r)=k_{a}(r / a)^{\gamma}, T(a)=T_{a}, T(b)=T_{b}\right\}$

Uniform Disk Made of a Homogeneous and Isotropic Material

$$
\frac{T^{\prime}(r)}{r}+T^{\prime \prime}(r)=0
$$

$$
\begin{aligned}
& T(r)=C_{2}+C_{1} \ln r \\
& C_{1}=\frac{T_{a}-T_{b}}{\ln a-\ln b} \\
& C_{2}=\frac{\ln a T_{b}-T_{a} \ln b}{\ln a-\ln b} \\
& T(r)=\frac{(-\ln b+\ln r) T_{a}+(\ln a-\ln r) T_{b}}{\ln a-\ln b} \\
& q(r)=\frac{k\left(-T_{a}+T_{b}\right)}{r(\ln a-\ln b)}
\end{aligned}
$$

Uniform Disk Made of a Power-Law-Graded Isotropic and Non-homogeneous Material

$$
\frac{(1+\gamma) T^{\prime}(r)}{r}+T^{\prime \prime}(r)=0
$$

$$
\begin{aligned}
& T(r)=-\frac{r^{-\gamma} C_{1}}{\gamma}+C_{2} \\
& C_{1}=\frac{\gamma a^{\gamma} b^{\gamma}\left(T_{a}-T_{b}\right)}{a^{\gamma}-b^{\gamma}} \\
& C_{2}=\frac{a^{\gamma} T_{a}-b^{\gamma} T_{b}}{a^{\gamma}-b^{\gamma}} \\
& T(r)=\frac{r^{-\gamma}\left(-a^{\gamma}\left(b^{\gamma}-r^{\gamma}\right) T_{a}+b^{\gamma}\left(a^{\gamma}-r^{\gamma}\right) T_{b}\right)}{a^{\gamma}-b^{\gamma}} \\
& q(r)=-\frac{a^{\gamma} b^{\gamma} r^{-1-\gamma}\left(\frac{r}{a}\right)^{\gamma} \gamma k_{a}\left(T_{a}-T_{b}\right)}{a^{\gamma}-b^{\gamma}}
\end{aligned}
$$

Variation of the temperature and heat flux along the radial coordinate for both convergent and divergent hyperbolic disks made of a SUS-304 $/ \mathrm{ZrO}_{2}$ functionally graded material is shown in Fig. 3 for different disk profile parameters. As the profile parameter goes from -1 towards 1 then the temperature changes much faster from the inner surface to the outer surface. The maximum heat flux occurs at the inner surface for both disk profile types, and decreases with increasing radial coordinate. Divergent disk profiles offer rapid changes in the heat flux at the vicinity of the inner surface while convergent disk profiles present much smoother variations in the radial direction.

Table 2. Differential equations and their solutions for hyperbolic disks $\left\{h(r)=h_{a}(r / a)^{m}\right.$ and $k(r)=k_{a}(r / a)^{\gamma}$,

$$
\left.T(a)=T_{a}, T(b)=T_{b}\right\}
$$

Hyperbolic Disk Made of a Homogeneous and Isotropic Material

$$
\frac{(1+m) T^{\prime}(r)}{r}+T^{\prime \prime}(r)=0
$$

$$
\begin{aligned}
& T(r)=-\frac{r^{-m} C_{1}}{m}+C_{2} \\
& C_{1}=\frac{m a^{m} b^{m}\left(T_{a}-T_{b}\right)}{a^{m}-b^{m}} \\
& C_{2}=\frac{a^{m} T_{a}-b^{m} T_{b}}{a^{m}-b^{m}} \\
& T(r)=\frac{r^{-m}\left(-a^{m}\left(b^{m}-r^{m}\right) T_{a}+b^{m}\left(a^{m}-r^{m}\right) T_{b}\right)}{a^{m}-b^{m}} \\
& q(r)=-\frac{a^{m} b^{m} m r^{-1-m} k_{o}\left(T_{a}-T_{b}\right)}{a^{m}-b^{m}}
\end{aligned}
$$

Hyperbolic Disk Made of a Power-Law-Graded Isotropic and Non-homogeneous Material

$$
\frac{(1+m+\gamma) T^{\prime}(r)}{r}+T^{\prime \prime}(r)=0
$$

$$
\begin{aligned}
& T(r)=-\frac{r^{-m-\gamma} C_{1}}{m+\gamma}+C_{2} \\
& C_{1}=\frac{(\gamma+m)\left(T_{a}-T_{b}\right) a^{\gamma+m} b^{\gamma+m}}{a^{\gamma+m}-b^{\gamma+m}} \\
& C_{2}=\frac{T_{a} a^{\gamma+m}-T_{b} b^{\gamma+m}}{a^{\gamma+m}-b^{\gamma+m}} \\
& T(r) \\
& =\frac{r^{-m-\gamma}\left(-a^{m+\gamma}\left(b^{m+\gamma}-r^{m+\gamma}\right) T_{a}+b^{m+\gamma}\left(a^{m+\gamma}-r^{m+\gamma}\right) T_{b}\right)}{a^{m+\gamma}-b^{m+\gamma}} \\
& q(r)=-\frac{a^{m+\gamma} b^{m+\gamma} r^{-1-m-\gamma}\left(\frac{r}{a}\right)^{\gamma}(m+\gamma) k_{a}\left(T_{a}-T_{b}\right)}{a^{m+\gamma}-b^{m+\gamma}}
\end{aligned}
$$



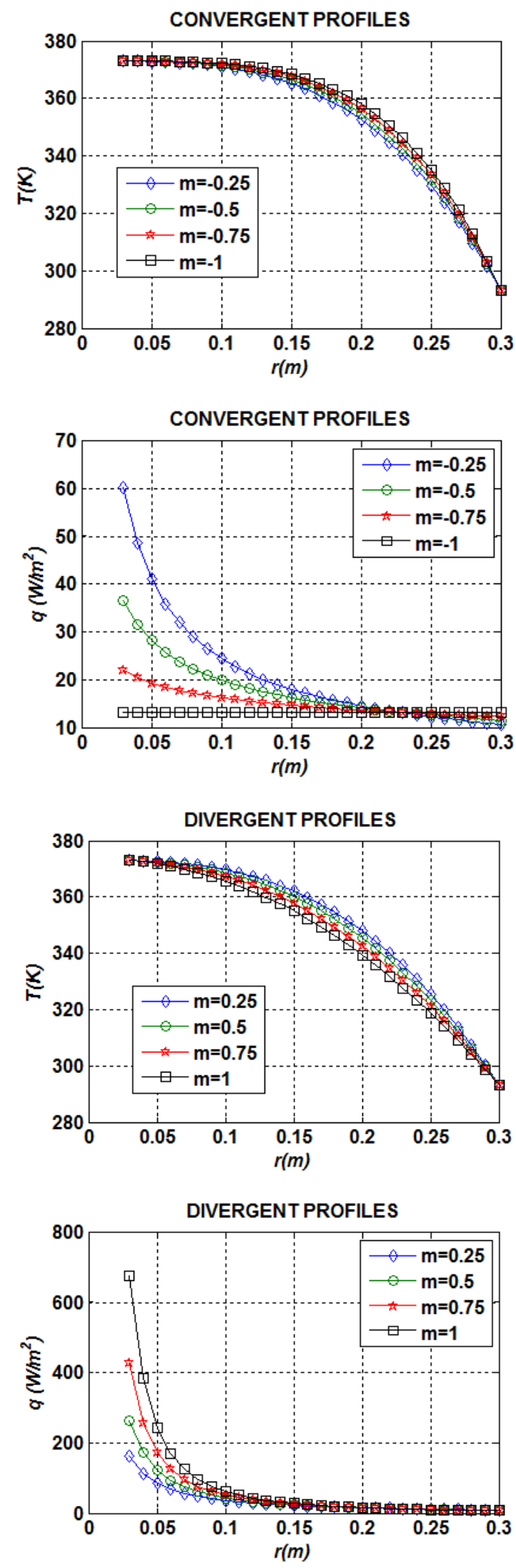

Fig. 3. Temperature and heat flux variation along the radial coordinate for convergent/divergent hyperbolic disks made of SUS-304 / $\mathrm{ZrO}_{2}$ FG material
To investigate the effects of the inhomogeneity parameters on the temperature distribution the followings are also assumed for any hypothetic FG-material: $\gamma=-3,-2,-1,-0.5,0.5,1,2,3$.

Effects of the inhomogeneity parameter on the temperature distribution for both divergent and convergent hyperbolic disks made of a hypothetic FG material are shown in Tables 3 and 4, and Figs. 4-7.

Temperature variation along the radial coordinate for special values of hypothetical inhomogeneity index $\gamma=0$, which means an isotropic and homogeneous material, and profile parameter $m=0$, which means a disk with unchanging thickness, are illustrated in Fig. 4.

From Figs. 4-7, and Tables 3-4, it is observed that the temperature distribution is strictly affected from the numerical values of both the inhomogeneity and profile parameters:

- For a chosen disk profile, either convergent or divergent, temperature variations with inhomogeneity indexes are observed clearly. For positive inhomogeneity indexes, almost a sudden temperature drop is observed at the vicinity of the inner surface for all types of profiles (Figs 4-5). This happens much faster if the divergent profiles are concerned.

Table 3. Temperature variation along the radial coordinate with divergent profile parameter and hypothetical inhomogeneity indexes.

\begin{tabular}{|c|c|c|c|c|}
\hline$r(m)$ & $\gamma=-1$ & $\gamma=-0.5$ & $\gamma=0.5$ & $\gamma=1$ \\
\hline \multicolumn{5}{|c|}{$m=1$ (divergent profile) } \\
\hline 0.03 & 373. & 373. & 373. & 373. \\
\hline 0.06 & 348.918 & 338.732 & 319.595 & 312.394 \\
\hline 0.1 & 331.17 & 320.084 & 303.962 & 299.465 \\
\hline 0.15 & 317.082 & 308.325 & 297.777 & 295.424 \\
\hline 0.2 & 307.087 & 301.315 & 295.187 & 294.01 \\
\hline 0.25 & 299.335 & 296.531 & 293.822 & 293.356 \\
\hline 0.3 & 293. & 293. & 293. & 293. \\
\hline \multicolumn{5}{|c|}{$m=0.75$ (divergent profile) } \\
\hline 0.06 & 353.551 & 343.917 & 323.873 & 315.766 \\
\hline 0.1 & 336.9 & 325.49 & 307.054 & 301.456 \\
\hline 0.15 & 322.083 & 312.449 & 299.571 & 296.423 \\
\hline 0.2 & 310.621 & 303.966 & 296.146 & 294.496 \\
\hline 0.25 & 301.145 & 297.794 & 294.22 & 293.544 \\
\hline \multicolumn{5}{|c|}{$m=0.5$ (divergent profile) } \\
\hline 0.06 & 357.675 & 348.918 & 328.556 & 319.595 \\
\hline 0.1 & 342.449 & 331.17 & 310.778 & 303.962 \\
\hline 0.15 & 327.268 & 317.082 & 301.889 & 297.777 \\
\hline 0.2 & 314.47 & 307.087 & 297.444 & 295.187 \\
\hline 0.25 & 303.194 & 299.335 & 294.778 & 293.822 \\
\hline \multicolumn{5}{|c|}{$m=0.25$ (divergent profile) } \\
\hline 0.06 & 361.203 & 353.551 & 333.554 & 323.873 \\
\hline 0.1 & 347.617 & 336.9 & 315.14 & 307.054 \\
\hline 0.15 & 332.446 & 322.083 & 304.797 & 299.571 \\
\hline 0.2 & 318.514 & 310.621 & 299.15 & 296.146 \\
\hline 0.25 & 305.436 & 301.145 & 295.535 & 294.22 \\
\hline
\end{tabular}


- The thermal behavior of the disk is much influenced from the inhomogeneity indexes than the profile parameters chosen between -1 through 1 (Tables 3 - 4). However, the inhomogeneity indexes which are much closer to $\gamma=0$ show marked variations with respect to the values of the profile parameter as in Figs. 6 and 7.

\section{Discussion and Conclusions}

In this study, the exact thermal analysis is conducted for a hollow hyperbolic disk made of either a homogeneous or an inhomogeneous radially functionally graded material. The simple power material gradation rule is used for the radial variation of the material properties and thickness variations to get closed-form solutions. Analytical compact expressions for the temperature and heat-flux distributions in the radial direction are proposed. These formulas may also be used directly in some thermal-related and optimization problems. They may also be served as sound benchmark results for advanced studies.

Table 4. Temperature variation along the radial coordinate with convergent profile parameter and hypothetical inhomogeneity indexes.

\begin{tabular}{|c|c|c|c|c|}
\hline$r(m)$ & $\gamma=-1$ & $\gamma=-0.5$ & $\gamma=0.5$ & $\gamma=1$ \\
\hline \multicolumn{5}{|c|}{$m=-1$ (convergent profile) } \\
\hline 0.03 & 373. & 373. & 373. & 373. \\
\hline 0.06 & 370.576 & 368.223 & 357.675 & 348.918 \\
\hline 0.1 & 364.829 & 359.714 & 342.449 & 331.17 \\
\hline 0.15 & 353.606 & 346.405 & 327.268 & 317.082 \\
\hline 0.2 & 337.893 & 330.644 & 314.47 & 307.087 \\
\hline 0.25 & 317.691 & 312.767 & 303.194 & 299.334 \\
\hline 0.3 & 293. & 293. & 293. & 293. \\
\hline \multicolumn{5}{|c|}{$m=-0.75$ (convergent profile) } \\
\hline 0.06 & 369.577 & 366.429 & 353.551 & 343.917 \\
\hline 0.1 & 362.538 & 356.297 & 336.9 & 325.49 \\
\hline 0.15 & 350.234 & 342.127 & 322.083 & 312.449 \\
\hline 0.2 & 334.387 & 326.703 & 310.621 & 303.966 \\
\hline 0.25 & 315.249 & 310.275 & 301.145 & 297.794 \\
\hline \multicolumn{5}{|c|}{$m=-0.5$ (convergent profile) } \\
\hline 0.06 & 368.223 & 364.111 & 348.918 & 338.732 \\
\hline 0.1 & 359.714 & 352.259 & 331.17 & 320.084 \\
\hline 0.15 & 346.405 & 337.444 & 317.082 & 308.325 \\
\hline 0.2 & 330.644 & 322.63 & 307.087 & 301.315 \\
\hline 0.25 & 312.767 & 307.815 & 299.334 & 296.531 \\
\hline \multicolumn{5}{|c|}{$m=-0.25$ (convergent profile) } \\
\hline 0.06 & 366.429 & 361.203 & 343.917 & 333.554 \\
\hline 0.1 & 356.297 & 347.617 & 325.49 & 315.14 \\
\hline 0.15 & 342.127 & 332.446 & 312.449 & 304.797 \\
\hline 0.2 & 326.703 & 318.514 & 303.966 & 299.15 \\
\hline 0.25 & 310.275 & 305.436 & 297.794 & 295.535 \\
\hline
\end{tabular}
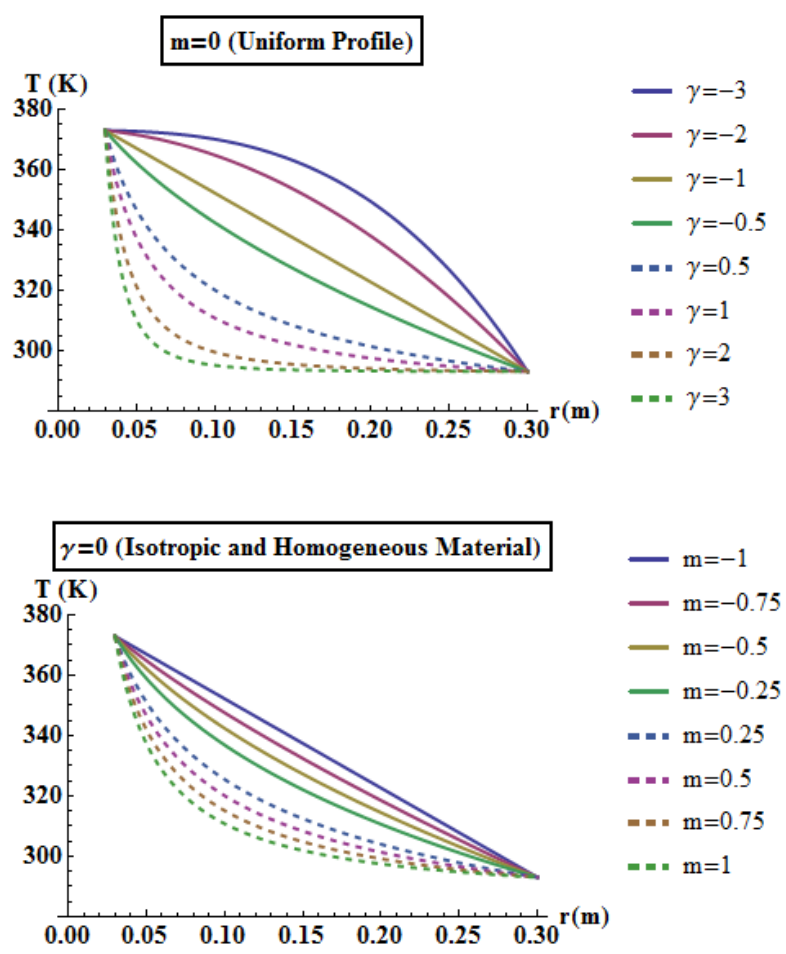

Fig. 4. Temperature variation along the radial coordinate for special values of profile parameter and hypothetical inhomogeneity index.
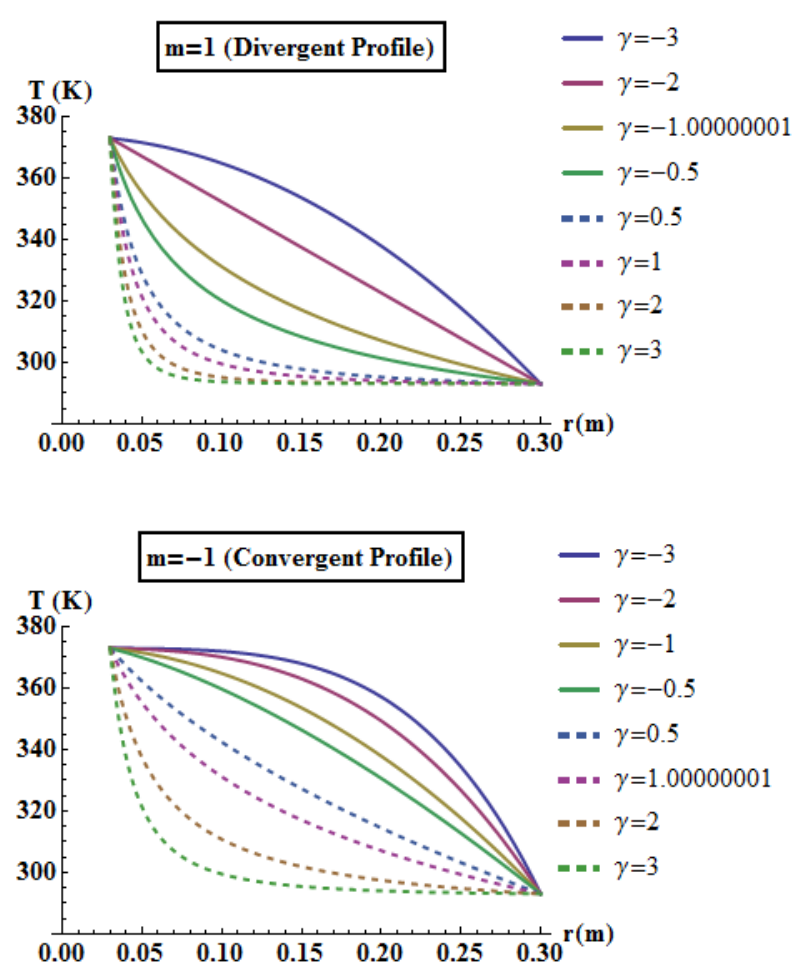

Fig. 5. Temperature variation along the radial coordinate with a divergent/convergent profile parameter and hypothetical inhomogeneity indexes. 

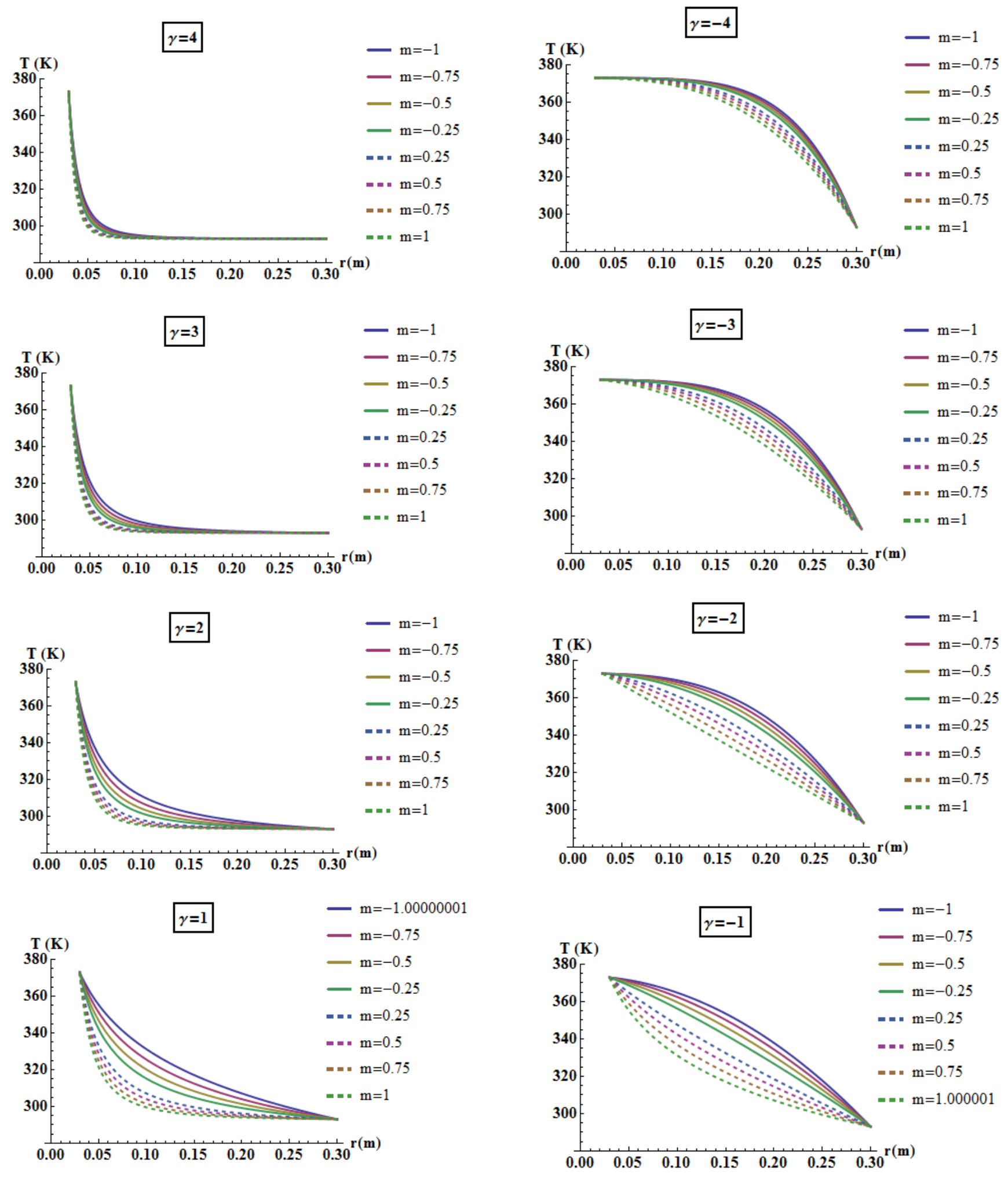

Fig. 6. Temperature variation along the radial coordinate with profile parameter and hypothetical positive inhomogeneity indexes.

Fig. 7. Temperature variation along the radial coordinate with profile parameter and hypothetical negative inhomogeneity indexes. 
A case study for a hyperbolic disk made of a physical metal-ceramic pair namely SUS-304 $/ \mathrm{ZrO}_{2}$ FG material is performed. Effects of the inhomogeneity parameter on the temperature distribution for both divergent and convergent hyperbolic disks made of hypothetic FG materials are also studied for different values of the profile parameter.

It may be noted that in the formulas presented in Table 2, when the sum of $m$ and $\gamma$ equals to integer zero, $m+\gamma=0$, the function becomes indefinite. In numerical computations, however, this may be get rid of by taking that sum as a real number closer to zero. This mini yet mighty tip is really works as seen from Figs. 5-7.

\section{Notations}

\begin{tabular}{|c|c|}
\hline$a$ & radius at the inner surface \\
\hline$b$ & radius at the outer surface \\
\hline$c_{p}$ & specific heat capacity $(J /(k g K))$ \\
\hline$C_{1}, C_{2}$ & integration constants \\
\hline $\boldsymbol{e}_{\boldsymbol{r}}, \boldsymbol{e}_{\theta}, \boldsymbol{e}_{z}$ & unit vectors in cylindrical coordinates \\
\hline$h$ & Thickness of the disk \\
\hline$k$ & thermal conductivity $(W /(m K))$ \\
\hline$m$ & Thickness parameter \\
\hline$q o r q_{r}$ & Heat flux component in radial direction \\
\hline$q$ & the rate of heat flux vector $\left(\mathrm{W} / \mathrm{m}^{2}\right)$ \\
\hline$\bullet$ & heat generation per unit volume \\
\hline$q_{\text {gen }}$ & \\
\hline$r$ & radial coordinate \\
\hline$t$ & time \\
\hline$T$ & temperature \\
\hline$\gamma$ & $\begin{array}{l}\text { inhomogeneity constant for simple- } \\
\text { power grading rule }\end{array}$ \\
\hline$\kappa=\frac{\rho c_{p}}{k}$ & thermal diffusion coefficient $\left(m^{2} / s\right)$ \\
\hline$\mu_{1}, \mu_{2}$ & $\begin{array}{l}\text { characteristic roots of the differential } \\
\text { equation }\end{array}$ \\
\hline$\rho$ & density $\left(k g / m^{3}\right)$ \\
\hline$\theta$ & circumferential coordinate \\
\hline$\nabla$ & gradient operator \\
\hline$\nabla^{2}=\Delta$ & Laplacian operator \\
\hline $\begin{array}{l}\frac{d}{d r}()=()^{\prime} \\
\text { subscripts }\end{array}$ & $\begin{array}{l}\text { derivative with respect to the radial } \\
\text { coordinate }\end{array}$ \\
\hline$a$, or $i$ & value at the inner surface \\
\hline$b$, or o & value at the outer surface \\
\hline
\end{tabular}

\section{References}

[1] U. Güven, and O. Altay, "Elastic-plastic solid disk with nonuniform heat source subjected to external pressure", International Journal of Mechanical Sciences, vol. 42(5), pp. 831-842, 2000.

[2] H. Jahed, and R. Shirazi, "Loading and unloading behaviour of a thermoplastic disc", International Journal of Pressure Vessels and Piping, vol. 78, pp. 637-645, 2001.

[3] V.S. Gogulwar, and K.C. Deshmukh, "An inverse quasistatic thermal stresses in an annular disc", Proceeding of ICADS, Narosa Publishing House, New Delhi, 2002.

[4] V.S. Kulkarni, and K.C. Deshmukh, "Thermal stresses in a thick annular disc, Journal of Thermal Stresses, vol. 31(4), pp. 331-342, 2008.

[5] M.S. Genç, G. Özşik, and H. Yapıcı, "A numerical study of the thermally induced stress distribution in a rotating hollow disc heated by a moving heat source acting on one of the side surfaces", Proceedings of the Institution of Mechanical Engineers, Part C: Journal of Mechanical Engineering Science, vol. 223(8), pp. 1877-1887, 2009.

[6] M.Z. Nejad, and A. Afshin, "Transient thermoelastic analysis of pressurized rotating disks subjected to arbitrary boundary and initial conditions", Chinese Journal of Engineering, Article ID 894902, 13 pages, 2014. http://dx.doi.org/10.1155/2014/894902

[7] M. Rattan, A. Kaushik, and N. Chamoli, "Steady state creep behavior of thermally graded isotropic rotating disc of composite taking into account the thermal residual stress", European Journal of Mechanics A/Solids, vol. 60, pp. 315-326, 2016.

[8] J. Kaur, P. Thakur, and S.B. Singh, "Steady thermal stresses in a thin rotating disc of finitesimal deformation with mechanical load", Journal of Solid Mechanics, vol. 8(1), pp. 204-211, 2016.

[9] P. Nayak, K. Saha, "Elastic limit angular speed of solid and annular disks under thermomechanical loading", International Journal of Engineering, Science and Technology, vol. 8(2), pp. 30-45, 2016.

[10] V. Yıldırım, "Heat-induced, pressure-induced and centrifugal-force-induced exact axisymmetric thermomechanical analyses in a thick-walled spherical vessel, an infinite cylindrical vessel, and a uniform disc made of an isotropic and homogeneous material", International Journal of Engineering \& Applied Sciences (IJEAS), vol. 9 (2), pp. 66-87, 2017.

[11] F. Vivio, and V. Vullo, "Elastic stress analysis of rotating converging conical disks subjected to thermal load and having variable density along the radius", International Journal of Solids and Structures, vol. 44, pp. 7767-7784, 2007. 
[12] V. Vullo, and F. Vivio, "Elastic stress analysis of non-linear variable thickness rotating disks subjected to thermal load and having variable density along the radius", International Journal of Solids and Structures, vol. 45, pp. 5337-5355, 2008.

[13] G.J. Nie, and R.C. Batra, "Stress analysis and material tailoring in isotropic linear thermoelastic incompressible functionally graded rotating disks of variable thickness", Compos. Struct., vol. 92, pp. 720-729, 2010.

[14] M. Garg, B.S. Salaria, and V.K. Gupta, "Effect of thermal gradient on steady state creep in a rotating disc of variable thickness", Procedia Eng., vol. 55, 542e547. 2013.

[15] E. Çetin, A. Kurşun, Ş. Aksoy, and M.T. Çetin, "Elastic stress analysis of annular bi-material discs with variable thickness under mechanical and thermomechanical loads", World Academy of Science, Engineering and Technology, International Journal of Mechanical, Aerospace, Industrial, Mechatronic and Manufacturing Engineering, vol. 8(2), pp. 288-292, 2014.

[16] O. Sayman, "Thermal stress analysis in an aluminum metal-matrix orthotropic disc", Journal of Reinforced Plastics and Composites, vol. 23, pp. 1473-1479, 2004.

[17] H. Çallıoğlu, M. Topçu, and G. Altan, "Stress analysis of curvilinearly orthotropic rotating discs under mechanical and thermal loading", Journal of Reinforced Plastics and Composites, vol. 24(8), pp. 831-838, 2005.

[18] H. Çallığlu, "Thermal stress analysis of curvilinear orthotropic rotating disks", Journal of Thermoplastic Composite Materials, vol. 20, pp. 357-369, 2007.

[19] F. Sen, and M. Sayer, "Elasto-plastic thermal stress analysis in a thermoplastic composite disc under uniform temperature using FEM", Mathematical and Computational Applications, vol. 11(1), pp. 31-39, 2006.

[20] G. Altan, M. Topçu, N.B. Bektaş, and B.D. Altan, "Elastic-plastic thermal stress analysis of an aluminum composite disc under parabolic thermal load distribution", J. Mech. Sci. Technol., 22(12), pp. 2318-2327, 2008.

[21] F. Mohammadi, A. Hadadian, and G.J. Singh, "Analytical solution of pressurized rotating composite disk under thermal loading", Proceedings of the World Congress on Engineering II: WCE 2010, London, U.K. (4 pages) 2010.

[22] M.T. Mustafa, S.M. Zubair, and A.F.M. Arif, "Thermal analysis of orthotropic annular fins with contact resistance: A closed-form analytical solution", Appl. Therm. Eng., vol. 31, pp. 937-945, 2011.

[23] G. Kansal, and M. Parvez, "Thermal stress analysis of orthotropic graded rotating discs", International Journal of Modern Engineering Research (IJMER), vol. 2(5), pp. 3381-3885, 2012.

[24] I.H. Stampouloglou, and E.E. Theotokoglou, "The radially nonhomogeneous thermoelastic axisymmetric problem", International Journal of Mechanical Sciences, vol. 120, pp. 311-321, 2017.

[25] W. Shang-Sheng, "Analysis on transient thermal stresses in an annular fin", Journal of Thermal Stresses, vol. 20, pp. 591-615, 1997.

[26] C.H. Chiu, and O.K.C. Chen, "Thermal stresses in annular fins with temperature dependent conductivity under periodic boundary condition", Journal of Thermal Stresses, vol. 25(5), pp. 475-492, 2002.

[27] M. Bayat, M. Saleem, B.B. Sahari, A.M.S. Hamouda, and E. Mahdi, "Thermo elastic analysis of a functionally graded rotating disk with small and large deflections", Thin-Wall Struct, vol. 45, pp. 677-691, 2007.

[28] S.A.H. Kordkheili, and R. Naghdabadi, "Thermoelastic analysis of a functionally graded rotating disk”, Composite Structures, vol. 79, pp. 508-516, 2007.

[29] A.G. Arani, M.R. Mozdianfard, Z.K. Maraghi, and A.R. Shajari, "Thermo-piezo-magneto-mechanical stresses analysis of FGPM hollow rotating thin disk", Int. J. Mech. Mater. Des., vol. 6, pp. 341-349, 2010.

[30] A.M. Afsar, J. Go, "Finite element analysis of thermoelastic feld in a rotating FGM circular disk", Appl. Math. Model, vol. 34, pp. 3309-3320, 2010.

[31] X.L. Peng, and X.F. Li, "Thermal stress in rotating functionally graded hollow circular discs", Composite Structures, vol. 92 (8), pp. 1896-1904, 2010.

[32] A. Kursun, M. Topçu, and T. Tetik, "Stress analysis of functionally graded disc under thermal and mechanical loads", ICM11, Engineering Procedia, vol. 10, pp. 2949 2954, 2011.

[33] J.F. Gong, P.J. Ming, L.K. Xuan, and W.P. Zhang, "Thermoelastic analysis of three-dimensional functionally graded rotating disks based on finite volume method", Proceedings of the Institution of Mechanical Engineers Part C., Journal of Mechanical Engineering Science, vol. 228(4), pp. 583-598, 2014.

[34] D. Gonczi, and I. Ecsedi, "Thermoelasic analysis of functionally graded hollow circular disk", Arch Mech Eng, vol. LXII, pp. 5-18, 2015.

[35] V. Yıldırım, "Thermomechanical characteristics of a functionally graded mounted uniform disc with/without rigid casing", The Journal of Aerospace Technology and Management (JATM), vol. 11: e2919 (25 pages), 2019.

[36] R. Chiba, "Stochastic thermal stresses in an FGM annular disc of variable thickness with spatially random heat transfer coefficients", Meccanica, vol. 44, pp. 159 176,2009

[37] M. Bayat, M. Saleem, B.B. Sahari, A.M.S Hamouda, and E. Mahdi, "Mechanical and thermal stresses in a functionally graded rotating disk with variable thickness due to radially symmetry loads", Int. Journal of Pressure Vessels and Piping, vol. 86, pp. 357-372, 2009.

[38] M. Bayat, B.B. Sahari, M. Saleem, A. Ali, and S.V. Wong, "Thermoelastic solution of a functionally graded 
variable thickness rotating disk with bending based on the first-order shear deformation theory", Thin-Wall Struct., vol. 47, pp. 568-582, 2009.

[39] M. Bayat, B.B. Sahari, M. Saleem, A.M.S. Hamouda, and J.N. Reddy, "Thermo elastic analysis of functionally graded rotating disks with temperaturedependent material properties: uniform and variable thickness", Journal of Mechanics and Materials in Design, vol. 5(3), pp. 263-279, 2009.

[40] M. Bayat, A.H. Mohazzab, B.B. Sahari, and M. Saleem, "Exact solution for functionally graded variablethickness rotating disc with heat source, Proceedings of the Institution of Mechanical Engineers-Part C., Journal of Mechanical Engineering Science, vol. 224(11), pp. 23162331, 2010.

[41] G. Arani, A. Loghman, A. Shajari, and S.A.R. Amir, "Semi-analytical solution of magneto-thermo-elastic stresses for functionally graded variable thickness rotating disks", J. Mech. Sci. Technol., vol. 24(10), pp. 2107-2117, 2010.

[42] M. Damircheli, and M. Azadi, "Temperature and thickness effects on thermal and mechanical stresses of rotating FG-disks", Journal of Mechanical Science and Technology, vol. 25(3), pp. 827-836, 2011.

[43] A. Hassani, M.H. Hojjati, G. Farrahi, and R.A. Alashti, "Semi-exact elastic solutions for thermo- mechanical analysis of functionally graded rotating disks", Composite Structures, vol. 93(12), pp. 32393251, 2011.

[44] N. Tutuncu, and B. Temel, "An efficient unified method for thermoelastic analysis of functionally graded rotating disks of variable thickness", Mechanics of Advanced Materials and Structures, vol. 20(1), pp. 38-46, 2013.

[45] M.E. Golmakani, "Large deflection thermoelastic analysis of shear deformable functionally graded variable thickness rotating disk", Composites: Part B, vol. 45, pp. 1143-1155, 2013.

[46] A. Kurşun, and M. Topçu, "Thermal stress analysis of functionally graded disc with variable thickness due to linearly increasing temperature load", Arab J Sci Eng, vol. 38, pp. 3531-3549, 2013.

[47] E. Mahdavi, R. AkbariAlashti, A.C. Darabi, and M. Alizadeh, "Linear thermoplastic analysis of FGM rotating discs with variable thickness", Iranian Journal Of Mechanical Engineering, vol. 14(2), pp. 73-87, 2013.

[48] M. Jabbari, M. Ghannad, and M.Z. Nejad, "Effect of thickness profile and FG function on rotating disks under thermal and mechanical loading", Journal of Mechanics, vol. 32(1), pp. 35-46, 2016. 